\title{
Cooperative Light-Induced Breathing of Soft Porous Crystals via Azobenzene Buckling
}

Simon Krause ${ }^{1,2 *}$, Jack D. Evans ${ }^{2}$, Volodymyr Bon ${ }^{2}$, Stefano Crespi ${ }^{1}$, Wojciech Danowski ${ }^{1}$, Wesley R. Browne ${ }^{1}$, Sebastian Ehrling ${ }^{2}$, Francesco Walenszus ${ }^{2}$, Dirk Wallacher ${ }^{3}$, Nico Grimm ${ }^{3}$, Daniel M. Többens ${ }^{3}$, Manfred S. Weiss $^{3}$, Stefan $\mathrm{Kaskel}^{2 *}$, Ben L. Feringa ${ }^{1 *}$

1) Centre for Systems Chemistry, Stratingh Institute for Chemistry, University of Groningen, Nijenborgh 4, 9747 AG Groningen, The Netherlands

2) Faculty of Chemistry and Food Chemistry, TU Dresden, Bergstrasse 66, 01062, Dresden, Germany

3) Helmholtz-Zentrum Berlin für Materialien und Energie, Hahn-Meitner-Platz 1, 14109, Berlin, Germany

Correspondence to:

Simon Krause: simon.krause@rug.nl

Stefan Kaskel: stefan.kaskel@tu-dresden.de

Ben L. Feringa: b.l.feringa@rug.nl

\begin{abstract}
Although light is a prominent stimulus for smart materials, the application of photoswitches as lightresponsive triggers for phase transitions of porous materials remains poorly explored. Here we incorporate an azobenzene photoswitch in the backbone of a metal-organic framework producing light-induced structural contraction of the porous network in parallel to gas adsorption. Light-stimulation enables non-invasive spatiotemporal control over the mechanical properties of the framework, which ultimately leads to pore contraction and subsequent guest release via negative gas adsorption. The complex mechanism of light-gated breathing is established by a series of in situ diffraction and spectroscopic experiments, supported by quantum mechanical and molecular dynamic simulations. Unexpectedly, this study identifies a novel light-induced deformation mechanism of constrained azobenzene photoswitches relevant to the future design of light-responsive materials.
\end{abstract}

\section{Introduction:}

The design of responsive porous materials, in which the porosity can be modulated externally and non-invasively by light to control adsorption, transport and release properties offers fascinating opportunities. Azobenzene (AB) molecular photoswitches ${ }^{1}$ (PS) undergo light-activated $E-Z$ isomerization and are frequently applied in lightresponsive actuators ${ }^{2}$ membranes $^{3}$, smart materials ${ }^{4}$, and single-molecule optical memories ${ }^{5}$. The design of responsive porous materials, in which the porosity can be modulated externally and non-invasively by light to control adsorption, transport and release properties offers fascinating opportunities. Pendent AB-switches grafted onto the backbone of porous metal-organic frameworks (MOFs) were demonstrated to reversibly control the separation and release of guest molecules by manipulating the porosity and host-guest interactions via photoswitching ${ }^{6-8}$. However, pendent AB switches occupy pore space, which could be used for guest inclusion, and lack cooperativity that would be highly beneficial for the efficiency and selectivity of adsorption processes. ${ }^{9,10}$ Soft porous crystals ${ }^{11}$ (SPCs) exhibit cooperative framework deformation dictated by the crystal structure. As a result, SPCs show adsorption phenomena such as gate-opening (pore expansion) ${ }^{12}$, breathing (pore contraction) $)^{13}$ and negative gas adsorption (NGA, gas release upon pore contraction) ${ }^{14}$ that show potential for improved diffusion ${ }^{15,16}$, storage ${ }^{17}$ and separation ${ }^{15}$ of gases and gas mixtures. Currently the contraction and expansion of the porous network of SPCs are primarily guest-induced and energetically driven via adsorption ${ }^{18}$. To date, the chemical modification of building blocks and framework topology are the dominant strategy to alter the guestresponsive behavior of SPCs ${ }^{19,20}$. The use of diarylethene PS in the framework backbone is a promising strategy to manipulate cooperative framework transitions ${ }^{21-24}$. However, the observed effects are very small, compared to the response due to guest-induced deformations of SPCs, and the initiation of massive framework deformations in SPCs by the application of both light- and guest-interactions is unprecedented. The large geometric change upon $E-Z$ isomerization of $\mathrm{AB}$ is expected to result in a much stronger framework deformation when incorporated in the framework backbone. Until now, photoswitching is observed to be either suppressed due to framework constraints $^{25,26}$ or causes irreversible bond-breaking and degradation of the extended framework ${ }^{27-30}$. The fundamental challenges of how to accommodate the large geometric change of $\mathrm{AB}$ upon $E-Z$ isomerization and establish photoinduced cooperative transitions, in the absence of framework disintegration, requires uncompromised/robust photoswitching, sufficient mechanical softness, enhanced porosity, and long-range order. Furthermore, it remains unexplored whether geometric constrains of framework-embedded PS might result in alternative photoswitching pathways, unknown for unconstrained molecular PS. 


\section{Modeling of molecular photoswitch and framework}

3

We selected the $49^{\text {th }}$ MOF material discovered at the University of Technology Dresden (DUT-49) ${ }^{31}$ as a blueprint for our new photoresponsive SPC design because of its ability to accommodate large changes in ligand configuration and framework structure without disintegration following substantial framework contraction ${ }^{14}$. The three dimensional (3D) framework of DUT-49 is based on the linkage of tetra-connective carbazole-based ligands to copper(II) dimers. By using (E)-9,9'-(diazene-1,2-diylbis(4,1-phenylene))bis( $9 H$-carbazole-3,6-dicarboxylic acid $\left((E)-\mathrm{H}_{4} \mathrm{dacdc}\right)$ we are able to establish the structurally related framework of DUT-163 which contains an AB functionality in the backbone (Figure 1).
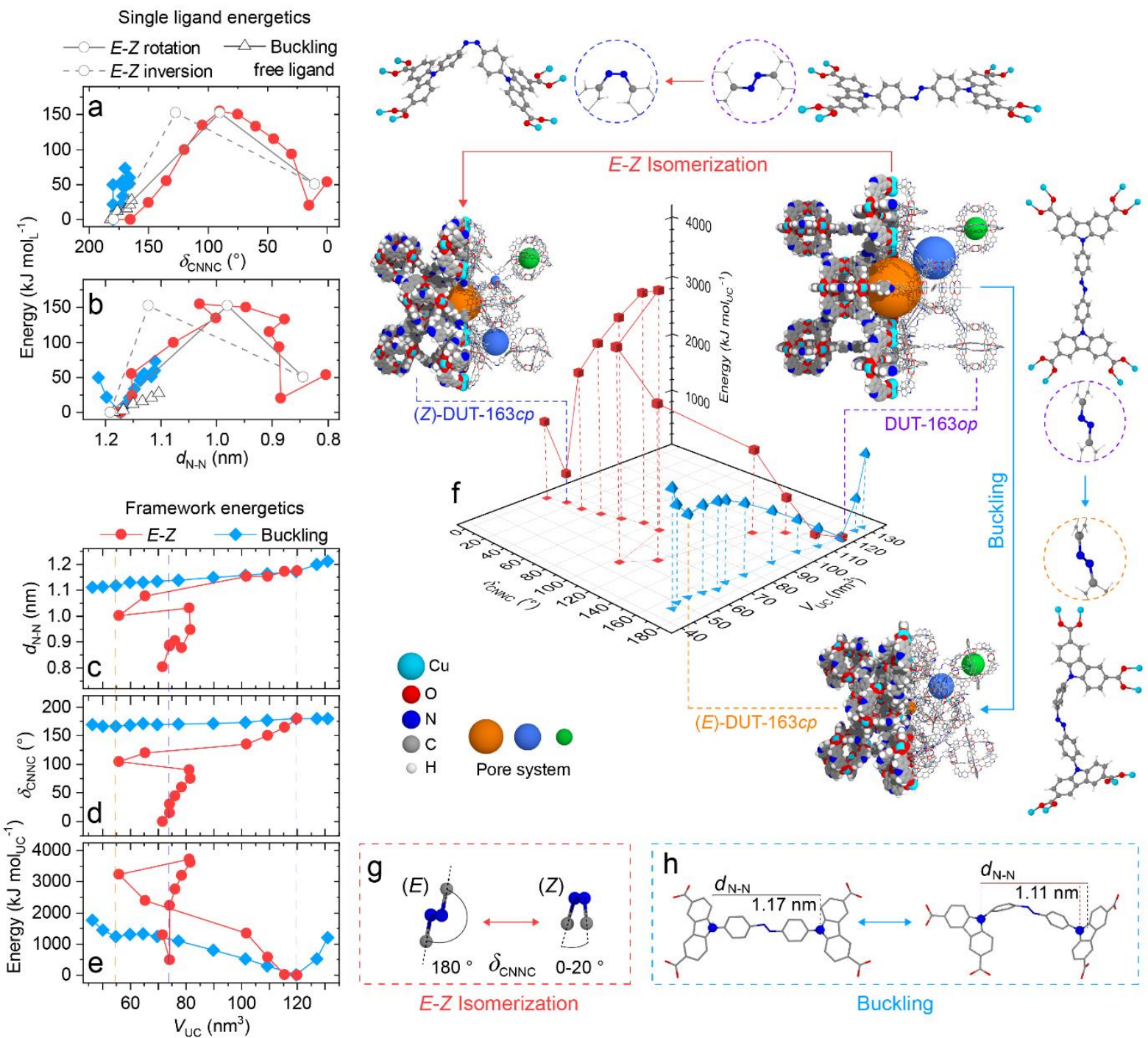

Figure 1. Energy landscapes of DUT-163. a,b Single ligand energetics as a function of $\delta_{\mathrm{CNNC}}(\mathbf{a})$ and $d_{\mathrm{N}-\mathrm{N}}(\mathbf{a})$ for framework constrained dacdc (red, blue) and unconstrained Me 4 dacdc (grey open symbols. c-d Framework contraction via E-Z isomerization (red squares) and ligand buckling (lblue diamonds) as a function of $d_{\mathrm{N}-\mathrm{N}}(\mathbf{c}), \delta \mathrm{CNNC}_{\mathrm{N}}(\mathbf{d})$ and energy (e), unit cell volumes of (Z)-DUT-163cp (blue), DUT-163op (purple), and (E)-DUT-163cp (orange) are given as dashed vertical lines. 3D energy landscape of DUT-163 (f) as a function of unit cell volume $\left(V_{\mathrm{UC}}\right), \delta_{\mathrm{CNNC}}$ with energy normalized to the $o p$ state including structures of (Z)-DUT-163cp (c), DUT-163op (d), and (E)-DUT-163cp (e) including the corresponding ligand structures and diaza-conformation in dashed circles. Schematics of $E-Z$ isomerization and ligand buckling including definition of $d \mathrm{~N}-\mathrm{N}$ and $\delta_{\mathrm{CNNC}}(\mathbf{g}, \mathbf{h})$.

We conducted density functional theory (DFT) simulations of $(E)-\mathrm{H}_{4} \mathrm{dacdc}$ and its methylester $\left((E)-\mathrm{Me}_{4} \mathrm{dacdc}\right)$, to probe the energetics upon buckling ${ }^{32}$ and $E$-Z-isomerization as a function of the distance between two AB-bridged carbazole-nitrogen atoms $\left(d_{\mathrm{N}-\mathrm{N}}\right)$ and the dihedral angle of the azo-unit $\left(\delta_{\mathrm{CNNC}}\right)$ (Figure $\left.1 \mathrm{~A}, \mathrm{~B}\right)$. Regardless of the $E-Z$ isomerization mechanism chosen (i.e. rotation or inversion ${ }^{33,34}$ ), the energy barrier of isomerization at the 
ground state is over five times larger than the barrier of buckling $(E)-\mathrm{Me}_{4} \mathrm{dacdc}$. This result is to be expected since buckling is a conformational change while $E$ - $Z$ isomerization involves the breaking of the azo $\pi$-bond in the ligand backbone. To investigate how the constraints imposed by the incorporation in a framework impact the energetics of $E$ - $Z$ isomerization and buckling, we computed the contraction mechanism of DUT-163 as a function of unit cell volume $\left(V_{\mathrm{UC}}\right)$ for buckling and $E-Z$ isomerization of the ligand by molecular dynamics (MD) simulations. Similar to the analysis of the unconstrained $\mathrm{Me}_{4}$ dacdc ligand, $E-Z$ isomerization of dacdc in DUT-163 exhibits a much larger energy barrier compared to the buckling transition (Figure $1 \mathrm{C}-\mathrm{E}$ ). However, the associated contraction mechanism of the DUT-163 framework exhibit two very different trajectories. The energy landscape of DUT-163 as a function of $V_{\mathrm{UC}}$ exhibits the global minimum at $V_{\mathrm{UC}}=120 \mathrm{~nm}^{3}$ corresponding to the open pore $(o p)$ state (DUT-163op) (Figure 1 F). A metastable state with buckled ligand in $E$ conformation at $V_{\mathrm{UC}}=54 \mathrm{~nm}^{3}$ is observed which is assigned to a contracted pore $(c p)$ state, further denoted as $(E)$-DUT-163cp (See supplementary video $1,2)$. To probe the framework energetics upon $E-Z$ isomerization of dacdc, we investigated the evolution of $V_{\mathrm{UC}}$ and the framework geometry as a function of $\varphi_{\mathrm{CNNC}}$ (See supplementary video 3,4). Interestingly, this energy landscape presents a local minimum at $V_{\mathrm{UC}}=74 \mathrm{~nm}^{3}$, which is assigned to a contracted framework with dacdc in $Z$-configuration, (Z)-DUT-163cp. The energy barrier for contraction $\left(E_{\text {op-cp }}^{\ddagger}\right)$ per unit cell (UC) between DUT$163 o p$ and $(E)$-DUT-163cp $\left(\mathrm{E}^{t} \mathrm{op}-(E) \mathrm{cp}=1250 \mathrm{~kJ}\right.$ moluc$\left.^{-1}\right)$ is ca. three times smaller compared to the barrier between DUT-163op and (Z)-DUT-163cp (E $\left.\mathrm{E}_{\mathrm{op}-(Z) \mathrm{cp}}^{t}=3900 \mathrm{~kJ} \mathrm{moluc}^{-1}\right)$ (Figure 1C,F). Based on this data it can be concluded that DUT-163 is theoretically able to undergo contraction via buckling or $E-Z$ isomerization, with buckling being the energetically more favorable mechanism at the ground state.

Photoswitching of the molecular ligand

$(E)-\mathrm{H}_{4}$ dacdc was synthesized using an established strategy (see Supplementary Information for details ${ }^{35}$. Upon irradiation at $365 \mathrm{~nm}(295-298 \mathrm{~K})$ we observed changes in the UV-Vis absorption, Raman (Figure 2) and ${ }^{1} \mathrm{H}$ nuclear magnetic resonance spectra (Supplementary Figure 7-10) of $(E)$ - $\mathrm{H}_{4} \mathrm{dacdc}$ and the corresponding $n$-butyl ester $\left((E)-n \mathrm{Bu}_{4} \mathrm{dacdc}\right)$, typical for light-induced $E-Z$ isomerization ${ }^{33,36}$.
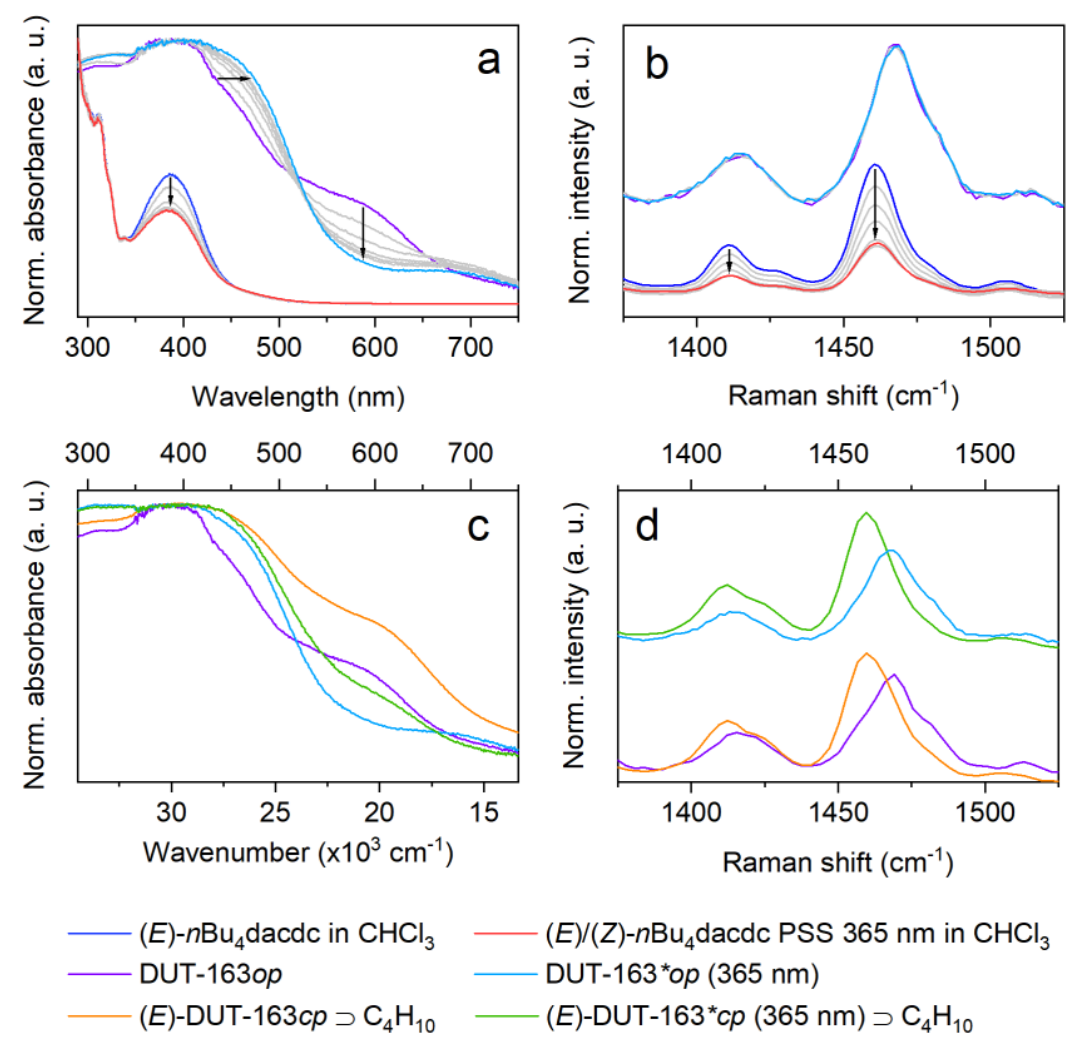

$$
\begin{aligned}
& \text { (E)/(Z)-nBu }{ }_{4} \text { dacdc PSS } 365 \mathrm{~nm} \text { in } \mathrm{CHCl}_{3} \\
& \text { DUT-163*op (365 nm) } \\
& \text { (E)-DUT-163* }{ }^{*} c p(365 \mathrm{~nm}) \supset \mathrm{C}_{4} \mathrm{H}_{10}
\end{aligned}
$$

Figure 2. In situ irradiation of ligand and MOF. a UV-Vis absorption spectra upon $365 \mathrm{~nm}$ irradiation of $n \mathrm{Bu}_{4} \mathrm{dacdc}$ in $\mathrm{CHCl}_{3}$ (red to blue) and DRUV-Vis absorption spectra of DUT-163 (purple to light blue), $\mathbf{b}$ Raman spectra of $n$ Bu4dacdc in $\mathrm{CHCl}_{3}$ (red to blue) and solid DUT-163 (purple to light blue) upon $365 \mathrm{~nm}$ irradiation, $\mathbf{c}$ DRUV-Vis absorption spectra of DUT-163 (purple) upon loading with $n$-butane (orange), $365 \mathrm{~nm}$ irradiation (light blue) and irradiation at $365 \mathrm{~nm}$ in the presence of $n$-butane (green), $\mathbf{d}$ and Raman spectra upon loading with 2-methylpropane and irradiation at $365 \mathrm{~nm}$. 
For both molecules, we observed a photostationary state (PSS) composed of a ca. 1:1 E-Z mixture at $293 \mathrm{~K}$. Upon irradiation at $455 \mathrm{~nm}$ the $Z$-isomer was partially reverted to a PSS comprising of ca. $75 \%$ of the $Z$-isomer. Thermal $Z-E$ isomerization was observed by heating above $338 \mathrm{~K}$ for over $5 \mathrm{~h}$ and the system showed excellent photochemical and thermal reversibility in solution.

\section{Synthesis of DUT-163}

The solvothermal reaction of $\mathrm{H}_{4}$ dacdc with $\mathrm{Cu}\left(\mathrm{NO}_{3}\right)_{2} \quad 3 \mathrm{H}_{2} \mathrm{O}$ in DMF at $80{ }^{\circ} \mathrm{C}$ yields DUT-163 as a brown microcrystalline powder with a mean crystal size of $2.6 \mu \mathrm{m}$ (Supplementary Figure 28). The single crystal structure of DUT-163 with cubic $F m \overline{3} m$ symmetry and cell dimensions of $a=49.240(6) \AA$ and a unit cell volume of $V_{\mathrm{UC}}=119386(41) \AA^{3}$ was determined by synchrotron-based single crystal X-ray diffraction (Supplementary Table 8 ), in line with the in silico optimized op structure. In DUT-163op, dacdc exhibits a linear (E)-configuration in which the AB-unit is disordered due to symmetrical restrictions (Supplementary Figure 24). The porous framework is characterized by a geometrical surface area, pore volume and pore diameters of GSA=5112 $\mathrm{m}^{2} \mathrm{~g}^{-1}$, $V_{\mathrm{p}}(\operatorname{sim})=3.2 \mathrm{~cm}^{3} \mathrm{~g}^{-1}$, and $d_{\mathrm{p}}=0.9-2.7 \mathrm{~nm}$, respectively which were simulated from the single crystal structure (Supplementary Figure 87). Desolvation of DUT-163 was achieved using supercritical carbon dioxide, a protocol previously described for DUT-4935. Permanent porosity was investigated by $\mathrm{N}_{2}$-adsorption experiments at $77 \mathrm{~K}$ from which a $V_{\mathrm{p}}$ of $2.84 \mathrm{~cm}^{3} \mathrm{~g}^{-1}$ (at $p / p_{0}=0.98$ ) was determined. The reduction in pore volume compared to the computed values might be based on crystal size effects previously observed for DUT- $49^{37}$.

\section{Spectroscopic analysis of structural contraction}

The light-responsiveness of DUT-163 was investigated by in situ PXRD, diffuse reflectance infrared Fourier transform spectroscopy (DRIFTS), solid state diffuse reflectance UV-Vis (DRUV-Vis) spectroscopy, and Raman spectroscopy experiments under dry nitrogen atmosphere with $365 \mathrm{~nm}$ irradiation at $293 \mathrm{~K}$. These conditions were previously found to promote $E-Z$ isomerization in solutions of both $n \mathrm{Bu}_{4}$ dacdc and $\mathrm{H}_{4}$ dacdc. Interestingly, we observed no significant changes in the Raman and DRIFT spectra as well as PXRD patterns of DUT-163 upon elongated $365 \mathrm{~nm}$ irradiation (Figure 2), indicating the absence of $E-Z$ isomerization of the ligand and structural contraction of the framework. This is further supported by nitrogen adsorption experiments at $77 \mathrm{~K}$, which showed no change in porosity after $365 \mathrm{~nm}$ irradiation (Supplementary Figure 29). However, upon $365 \mathrm{~nm}$ irradiation, we observed a bathochromic shift of the absorption in the DRUV-Vis spectrum of DUT-163 corresponding to the ABfunctionality, and decrease of the signal assigned to the absorption of the $\mathrm{Cu}^{2+}$-dimer at (Figure $2 \mathrm{~A}$ ). These changes were found to be irreversible upon irradiation at $455 \mathrm{~nm}$ (Supplementary Figure 75). Rather than $E$ - $Z$ isomerization which would cause pronounced changes in the PXRD patterns as well as Raman and DRIFT spectra, we propose a photoinduced charge transfer from the $\mathrm{AB}$-functionality to the $\mathrm{Cu}^{2+}$ site, which is reported in other metal-AB-complexes ${ }^{38}$. The absence of changes upon irradiation in the DRUV-Vis spectrum of DUT-49 (Supplementary Figure 71), which does not contain an oxidizable AB-unit, supports such mechanism. Furthermore, spin-flip DFT calculations on $\mathrm{Cu}_{2}$ dacdc indicate that the HOMO is located on the AB backbone while the LUMO is localized on the $\mathrm{Cu}^{2+}$-dimer, which would support the feasibility of photoinduced charge transfer (Supplementary Figure 94).

\section{Adsorption-induced structural contraction}

Methane $(111 \mathrm{~K})$ and $n$-butane $(298 \mathrm{~K})$ adsorption isotherms of DUT-163, in contrast to nitrogen (77 K), exhibit hysteresis and NGA steps (Supplementary Figure 30), evidence for adsorption-induced contraction well studied in DUT-49 and related frameworks ${ }^{39-42}$. Similar behavior is observed upon adsorption of 2-methylpropane (MP) in the temperature range of 262 - $297 \mathrm{~K}$ with no contraction in the range of 299 - $307 \mathrm{~K}$ (Supplementary Figure 31Supplementary Figure 39). This observation is in agreement with previous reports of DUT-49, where NGA and contraction was only observed in a narrow range of temperature and pressure for a given gas ${ }^{43}$. In situ PXRD in parallel to adsorption of $n$-butane at $298 \mathrm{~K}$ (Supplementary Figure 41) shows that DUT-163op undergoes a phase transition to $(E)$-DUT-163cp. Upon adsorption of MP at $262 \mathrm{~K}$ (Supplementary Figure 40) the $o p$ - $c p$ transition occurs at a relative pressure of 0.14 and reopening is observed at a relative pressure beyond 0.6. The phase transition is accompanied by a strong reduction in diffraction intensity which is again increased at higher relative pressure upon reopening, in line with previous studies on related solids ${ }^{14,40,41}$. The crystal structure of $(E)$-DUT$163 c p$ could be determined by Rietveld refinement using PXRD data recorded at $262 \mathrm{~K}$ and $p / p_{0}=0.25$ of $\mathrm{MP}$ (Supplementary Figure 69). The experimental structure is in good agreement with the previously described simulation of (E)-DUT-163cp (Figure 1) demonstrating the validity of the MD simulations of this system. To 
further analyze the adsorption behavior of DUT-163 we simulated MP isotherms for (E)-DUT-163cp and DUT$163 o p$ at $261 \mathrm{~K}, 296 \mathrm{~K}, 300 \mathrm{~K}$ and $330 \mathrm{~K}$ by grand canonical Monte Carlo (GCMC) methods. This approach allows the characterization of thermodynamic conditions for adsorption-induced contraction upon MP adsorption using the grand canonical ensemble ${ }^{43}$. Interestingly, adsorption-induced contraction becomes thermodynamically unfeasible at increasing temperature (Supplementary Figure 89) due to decreasing adsorption interactions. This explains the experimental upper temperature limit around $299 \mathrm{~K}$ for contraction upon adsorption of MP in DUT163.

\section{Adsorption- and photo-induced structural contraction}

Although light-driven contraction via $E-Z$ isomerization was not observed in guest-free DUT-163, we reasoned that additional adsorption interactions might help to stabilize a contracted (Z)-DUT-163 $\mathrm{cp}$ state and trigger a structural response by parallel application of light and gas adsorption. In initial experiments we recorded MP physisorption isotherms of two individual samples of DUT-163 in the temperature range of 307-295 K for which one sample was irradiated at $365 \mathrm{~nm}$ throughout the whole experiment, while the other was kept under light exclusion. Still, no differences between the adsorption isotherms of irradiated and non-irradiated DUT-163 samples could be detected, neither in the temperature range above nor below 299 K ((Figure 3 A-C, Supplementary Figure 31-Supplementary Figure 39) indicating the absence of light-induced contraction of the porous material in this experimental setup.
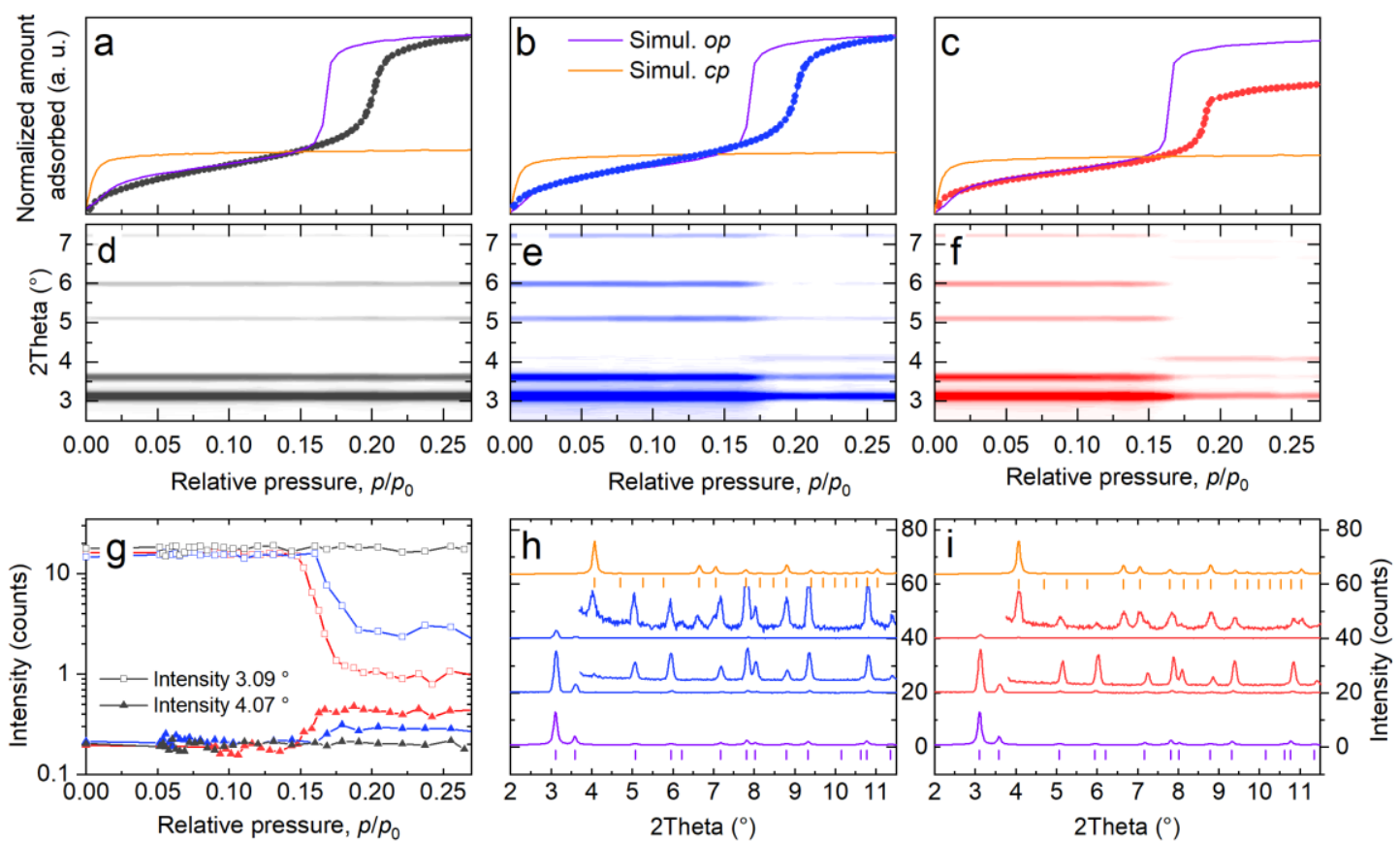

Figure 3. In situ diffraction analysis. a-c MP adsorption isotherms at $299 \mathrm{~K}$ (a), $299 \mathrm{~K}$ upon irradiation with $365 \mathrm{~nm}$ (b) and $295 \mathrm{~K}$ (c) in comparison to simulated isotherms for DUT-163op (purple line) and (E)-DUT-163cp (orange line) at $300 \mathrm{~K}$ (a,b) and $296 \mathrm{~K}$ (c). d-g Contour plots of in situ PXRD data recorded in parallel to adsorption of MP at $300 \mathrm{~K}$ (grey) (d), at $300 \mathrm{~K}$ with $365 \mathrm{~nm}$ irradiation (blue) (e) and at $296 \mathrm{~K}$ (red) (f) and corresponding evolution of peak intensity of the (111) reflection of DUT-163op (filled squares) and (E)-DUT-163cp (open triangles) at $3.1^{\circ}$ and $4.02^{\circ}$, respectively (g). h,i Selected PXRD patterns and magnified wide angle region as inset before (middle bottom) and after (middle top) structural contraction at $299 \mathrm{~K}$ upon irradiation with $365 \mathrm{~nm}$ (h) and at $295 \mathrm{~K}$ without irradiation (i) in comparison to simulated PXRD patterns and peak positions for DUT-163op (purple) and (E)-DUT-163cp (orange).

However, the large sample amount (>10 mg) required for accurate gas adsorption experiments can cause undersaturation of the whole bulk solid with light. In addition, adsorption experiments only provide little information on structural transitions that potentially occur only for a small part of the bulk sample. In order to explore in more detail, the light-responsive structural behavior of DUT-163 in parallel to the adsorption of MP we designed an in situ experimental setup that allows exposing small sample amounts $(<0.2 \mathrm{mg})$ to defined gas pressure while irradiating with light of defined wavelengths under isothermal conditions and tracking structural transitions by synchrotron-based PXRD. In a series of experiments, we probed the adsorption-induced structural transition upon MP adsorption at $296 \mathrm{~K}$ and $300 \mathrm{~K}$ (Figure 3f). 
At $296 \mathrm{~K}$ and relative pressure of $0.15-0.16$, we observed an $o p-c p$ transition (Figure 3F), demonstrating the ability to generate, observe, and identify the nature of adsorption-induced structural changes in DUT-163 with the new setup. In a second experiment we raised the temperature to $300 \mathrm{~K}$, beyond the upper temperature limit for adsorption-induced contraction. As expected, no structural contraction is observed (Figure 3D) in line with the gas adsorption experiments at $299 \mathrm{~K}$ (Figure 3a). In a third experiment, we used the same conditions (300 K) on the same sample but this time irradiated only parts of the sample, throughout the whole adsorption process, with $365 \mathrm{~nm}$ light. Interestingly, at a relative pressure of $0.17-0.18$ we observed a strong decrease in diffraction intensity at $3.09^{\circ} 2 \theta$ and appearance of new peaks at $4.07^{\circ}$ and $6.66^{\circ} 2 \theta$ (Figure $3 \mathrm{E}, \mathrm{H}$ ), which we can assign to the formation of $(E)$-DUT-163 cp. Reversible reopening of the structure was not observed in the investigated pressure range but is expected to occur at increasing relative pressures similar to the experiment conducted at $262 \mathrm{~K}$. Repetition of the experiments at $300 \mathrm{~K}$ on three individual samples confirmed the initial observations and the lightresponsive behavior (Supplementary Figure 48-Supplementary Figure 52). In all experiments, the temperature recorded in close proximity to the sample was stable at $300 \mathrm{~K}$, with fluctuations below $\pm 0.2 \mathrm{~K}$. We observed no change in the diffraction patterns of (E)-DUT-163cp upon irradiation with $365 \mathrm{~nm}$ and $455 \mathrm{~nm}$ light (Supplementary Figure 45), reflecting the absence of light-induced $c p$-op transition by potential Z-E photoisomerization. In one experiment, $365 \mathrm{~nm}$ irradiation was applied only in the relative pressure range of 0.16$0.28,1 \mathrm{~min}$ before the $o p-c p$ transition occurred, demonstrating that prolonged irradiation is not essential and the light application allows for temporal control of the process.

\section{Spatial control over light-induced contraction}

Because only a $6 \mathrm{~mm}$ length section of the sample-filled capillary was irradiated in the experiments described above, we performed an axial PXRD scan along the capillary to determine the spatial phase composition (Figure 4).
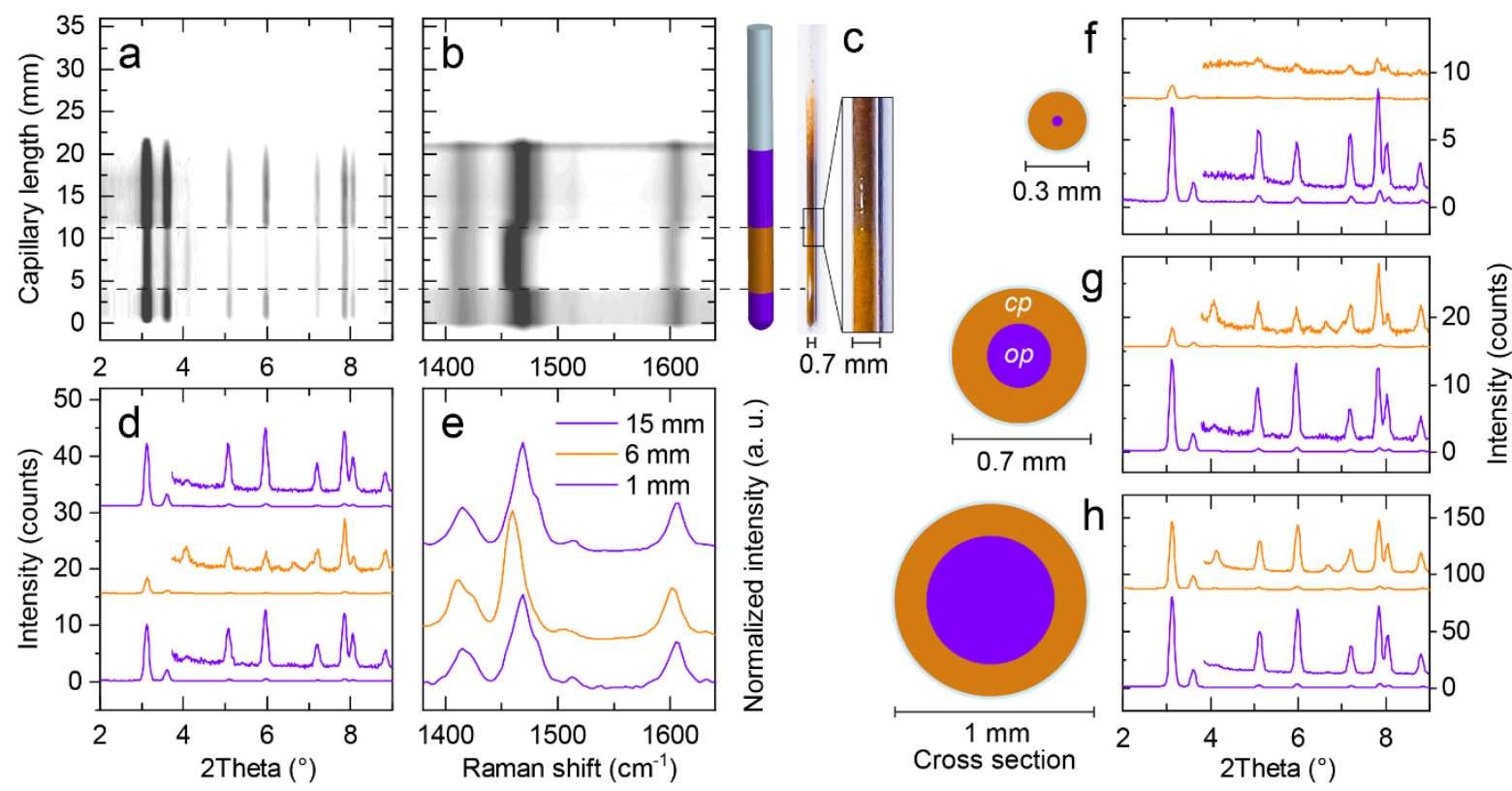

Figure 4. Analysis of local photo-induced transition. a-e Axial PXRD (a) and Raman scan (b) of a capillary with a diameter of $0.7 \mathrm{~mm}$, image and illustration of the investigated capillary (c), selected PXRD patterns at $1 \mathrm{~mm}$ (bottom), $6 \mathrm{~mm}$ (middle), and $13 \mathrm{~mm}$ (top) (d), Raman spectra at selected positions (e), f-h PXRD patterns of non-irradiated and irradiated areas for capillaries with diameters of $0.3 \mathrm{~mm}(\mathbf{f}), 0.7 \mathrm{~mm}(\mathbf{g})$, and $1 \mathrm{~mm}(\mathbf{h})$. Irradiated regions are marked in orange, non-irradiated areas in purple, inset enlarged patterns in $\mathbf{f}-\mathbf{h}$ of 2 Theta range $3.8-9^{\circ}$ represent 20 times magnification.

Only DUT-163 powder in the irradiated area exhibited structural contraction, supporting that light is indeed the trigger for the transition and demonstrating the spatial applicability of light-initiated contraction in DUT-163 (Figure 4 A,D). However, in all irradiation experiments, the residual op phase detected by PXRD indicates that only part of the sample undergoes a contraction. As the dense packing and high absorptivity of DUT-163 in the range of 200-600 nm can filter the light stimulus, we tested the light penetration depth by analyzing DUT-163filled quartz capillaries with diameters of $0.3 \mathrm{~mm}, 0.7 \mathrm{~mm}$ and $1 \mathrm{~mm}$ (wall thickness $0.01 \mathrm{~mm}$ ) (Figure $4 \mathrm{~F}-\mathrm{H}$ ). We observed that $88 \%(0.3 \mathrm{~mm}), 79 \%(0.7 \mathrm{~mm})$, and $26 \%(1 \mathrm{~mm})$ of the bulk sample underwent contraction, 
estimated from the change in intensity of the (111) reflection of DUT-163op at $2 \theta=3.09^{\circ}$. Thus, we evaluated that the penetration depth of the applied LED light is in the range of $0.1-0.15 \mathrm{~mm}$ for a non-compressed sample bed of DUT-163 powder. Although a more powerful light source might initiate contraction in a denser or thicker sample bed, the applied low power $15 \mathrm{~mW}$ LED used for irradiation in these experiments is sufficient to trigger structural contraction in microscopic or nanoscopic single crystals or thin films.

\section{In situ Adsorption-Light Spectroscopic experiments}

Although the presence of diffraction peaks of $(E)$-DUT-163cp clearly shows that the MP adsorption-induced structural transition at low temperature $(262 \mathrm{~K}, 296 \mathrm{~K})$ is comparable to the light-gated transition at higher temperature $(300 \mathrm{~K})$, the strong loss in crystallinity upon contraction might conceal the formation of states such as (Z)-DUT-163cp. We thus complemented PXRD experiments by in situ DRUV-Vis, Raman, and DRIFT spectroscopy upon adsorption of $n$-butane at $295 \mathrm{~K}$ with and without irradiation (Figure 2). In contrast to irradiation of guest free DUT-163, adsorption-induced contraction altered the spectra in all experiments strongly (Supplementary Figure 73) similar to experiments carried out on DUT-49 (Supplementary Figure 70). DUT-163 samples exhibited the same contraction behavior with and without $365 \mathrm{~nm}$ irradiation (Supplementary Figure 75), supporting the crystallographic findings: DUT-163op transforms into (E)-DUT-163cp via ligand buckling in response to adsorption-induced structural contraction, that can be promoted by $365 \mathrm{~nm}$ irradiation. Notably, this occurs at higher adsorption temperature at which adsorption stress alone is insufficient to trigger a contraction. Light-induced $E-Z$ isomerization of dacdc and the formation of (Z)-DUT-163cp is based on our experimental observations excluded as the underlying mechanism for the light-responsive framework contraction.

\section{Modeling of photoexcited state}

To postulate a mechanism of how irradiation can promote contraction via buckling we computed the photoexcitation process of framework-constrained dacdc. Ligand geometries upon buckling were extracted from the MD simulations of the DUT-163 contraction (Figure 1F). The energy landscapes of the ground state $\mathrm{S}_{0}$ and excited states $\mathrm{S}_{1}$ and $\mathrm{S}_{2}$ for $\mathrm{H}_{4}$ dacdc were determined by TD-DFT calculations as a function of $d_{\mathrm{N}-\mathrm{N}}$ distances and $\alpha_{\mathrm{CNN}}$ angles. ${ }^{44}$ (Figure 5).
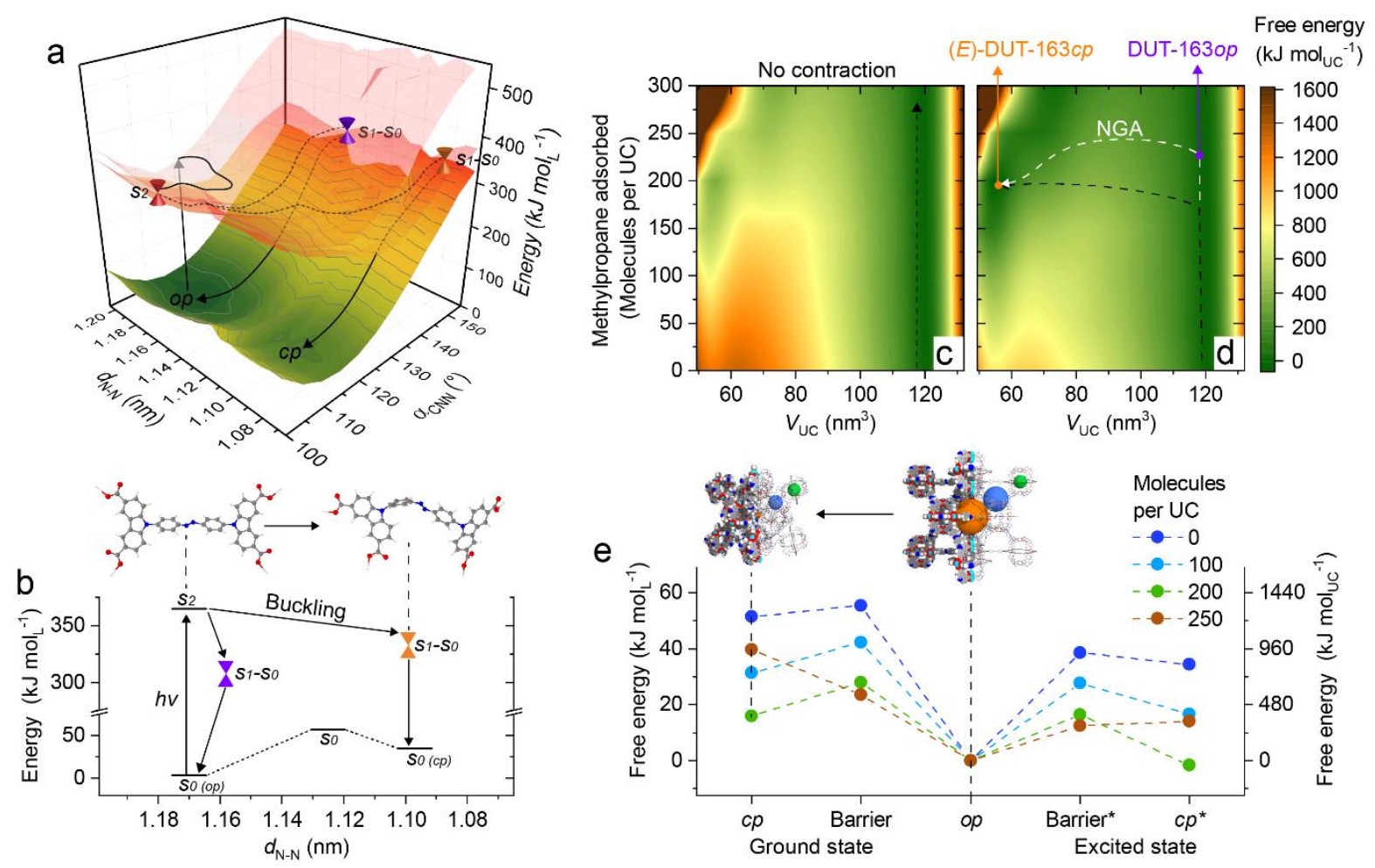

Figure 5. Energy landscape of dacdc and DUT-163. a Energy landscape in 3 dimensions (a) and a 2D projection (b) of groundstate $\mathrm{S}_{0}$ and excited state $\mathrm{S}_{1}$ and $\mathrm{S}_{2}$ of $\mathrm{H}_{4}$ dacdc as a function of $d_{\mathrm{N}-\mathrm{N}}$. Crossing points and potential energy trajectories are marked as cones and dashed lines, respectively. Ligand conformations are given for the $o p$ and $c p$ state. c-e Energy 
landscape at different loadings of MP at $300 \mathrm{~K}$ as contour plots of DUT-163 (c) and DUT-163* (d) as a function of unit cell volume normalized to the energy of the op state; $\mathbf{e} 2 \mathrm{D}$ energy diagram for selected loadings of the $o p$ and $c p$ state for DUT-163 and DUT-163* including transition barriers. Trajectories in D for contraction without and with NGA transition is indicated as black and white lines, respectively.

Interestingly, the energy landscape of framework-constrained dacdc exhibits a trajectory that promotes the formation of a metastable buckled state in $E$-conformation with reduced $d_{\mathrm{N}-\mathrm{N}}$ and $\varphi_{\mathrm{CNNC}} \approx 175^{\circ}$ (Figure $5 \mathrm{~A}$ ) which represents a novel photochemical deformation so far unexplored in $\mathrm{AB}$. This channel resembles the one promoting the nonproductive deactivation of the $A B$ excited state, after population of $S_{2}{ }^{33,45}$. Although this analysis shows that both buckling or $E-Z$ isomerization provide potential mechanism for light-induced contraction, the majority of individual ligands in the lattice of a single crystal would need to be instantaneously excited and transformed to drive cooperative structural contraction by light. The potential lack of collective excitation, supposedly short lifetimes of the excited states of individual ligands, and undersaturation of the bulk sample by the applied light source thus prevent observable photo-initiated contraction of guest-free DUT-163. However, upon adsorption of MP the energy landscape of DUT-163 changes. MD simulations of DUT-163 at $300 \mathrm{~K}$ at different loadings of MP per UC show that the energy difference between DUT-163op and (E)-DUT-163cp as well as the contraction barrier is reduced with increasing loading of MP. The contraction barrier is decreased by $50 \%$ at a loading of 200 molecules of MP per UC, at which the pores of (E)-DUT-163cp $\supset 200 i-\mathrm{C}_{4} \mathrm{H}_{10}$ are saturated. Following increasing loading, $(E)$-DUT-163cp is severely destabilized due to the lower $V_{\mathrm{p}}$ that cannot accommodate enhanced amounts of MP. Although (E)-DUT-163cp $\supset 200 i-\mathrm{C}_{4} \mathrm{H}_{10}$ still represents a metastable state at the highest level of adsorptioninduced stabilization, the reduction in contraction-barrier can make this state more prone to light-induced contraction.

To probe the response of DUT-163 upon irradiation we modeled an excited state of DUT-163 (DUT-163*) using established classical potentials that resemble the mechanics of dacdc in a biradical or zwitterionic state (further denoted to as dacdc*). This state is also a good mechanical representation of the previously described photooxidized state upon charge transfer between $\mathrm{AB}$ and $\mathrm{Cu}^{2+}$. We investigated the free energy landscape of guest free DUT-163* upon loading with MP using the same MD method applied in the analysis of the DUT-163 ground state. Interestingly, guest free DUT-163* is found to exhibit a much lower barrier for contraction compared to DUT-163. The breakage of $\pi$-conjugation in the ligand backbone of DUT-163* is the origin for this softening, which is also found to occur in chemically modified DUT-49-type frameworks ${ }^{42}$. It is well reflected by the simulated bulk modulus of $4.8 \mathrm{GPa}$ for guest free DUT-163 and 4.1 GPa for guest free DUT-163*, respectively. Because DUT-163* is mechanically softer compared to DUT-163 it is more susceptible to adsorption stress. In fact, at a loading of 200 molecules MP per UC, (E)-DUT-163* $c p \supset 200 i-\mathrm{C}_{4} \mathrm{H}_{10}$ was found to be the thermodynamically stable state with a reduction in contraction barrier of $42 \%$ compared to DUT-163כ200i- $\mathrm{C}_{4} \mathrm{H}_{10}$ under the same conditions. Although in the model of DUT-163* all ligands are simultaneously in the excited state, which might not occur in a real crystal, even partial photoexcitation or -oxidation is expected to soften the framework of DUT-163 significantly. The nature of the mechanism triggering the softening of the structure can hence be hypothesized to be buckling of the chromophore either via an excited state pathway, as described in Figure $5 \mathrm{~A}$, a photooxidation of the azo group by the $\mathrm{Cu}^{2+}$ clusters (Supplementary Figure 94), or a combination of both. Additional adsorption interactions lower the barrier for contraction and initiating a light/adsorptioninduced cooperative contraction of the crystal.

This dual-stimulus approach provides several advantages over purely light- or adsorption-induced transitions: photoexcitation allows the framework to respond to lower adsorption-induced stress levels and even drive contraction of a metastable state beyond the upper temperature limit of adsorption-induced contraction. The observed contraction results in gas release by NGA in an extended temperature range with a potentially increased magnitude. In addition, it allows to trigger NGA by a physical stimulus that specifically interacts with the framework. Thus, this process can be applied orthogonally to other non-radiative processes and other chemical or physical stimuli. Finally, it provides the possibility to spatially and temporarily control the release of gas via NGA using light as a physical trigger.

\section{Conclusion}

In conclusion, we show for the first time a cooperative structural transition of a SPC by combined application of light and adsorption-stress. Although DUT-163 was initially designed for contraction via $E$ - $Z$ isomerization of the AB-backbone, this process was ruled out by a combination of in situ experiments and extensive computation. Instead, the contraction mechanism is based on a buckling process, previously unknown for molecular ABs, and 
highlights the impact of framework-constraint on the behavior of photo-switches. In DUT-163 photoexcitation causes framework softening, allowing to drive structural contraction at reduced adsorption stress levels. The effect is reproducible under different conditions and allows for spatial and temporal control over the framework contraction by light. As such, light-responsive gas release by NGA can be locally and temporarily activated in DUT-163 for the use in nanoscopic pneumatic systems and gas-releasing devices. The postulated mechanism not only demonstrates a novel switching transition in $\mathrm{AB}$ and an unexplored way of initiating structural transitions in SPCs, it provides a novel strategy to physically alter the mechanical properties of extended molecular frameworks without the application of chemical functionalization, potentially allowing such frameworks to respond to other forms of stimuli such as electric or magnetic fields, temperature, or mechanical pressure which would result in a novel class of mechanical nanoscopic actuators. Furthermore, we believe the findings of this study go beyond the discovery of a novel mechanism of a light-induced cooperative transition in a SPC. Over the past years, many ABdoped materials were shown to exhibit light-induced changes of their properties upon irradiation ${ }^{23,46}$. In the vast majority of cases $E$-Z-photoisomerization was postulated as the primary origin for the observed behavior. The present study clearly illustrates that framework- or matrix-constrained photoswitches can exhibit properties and states very different to the unrestricted single molecular analogue. We conclude that photochemical properties of self-assembled systems are also governed by the structure and nature of the assembly beyond the properties of the molecular building blocks. In-depth analysis of these effects will lead to new design principles and novel properties of smart materials which may give rise to unexpected responsive behavior.

\section{Acknowledgements:}

The authors would like to thank the Helmholtz Zentrum Berlin for continuous support in establishing in situ PXRD infrastructure and allocation of beamtime. We would like to thank the Center for Information Technology of the University of Groningen for their support and for providing access to the Peregrine high-performance computing (HPC) cluster. Additional HPC platforms were provided by a GENCI grant (A0070807069) and the Center for Information Services and High Performance Computing (ZIH) at TU Dresden.

\section{Funding:}

S. Krause, B. L. F., and J. D. E. acknowledge financial support from the Alexander von Humboldt Foundation. S. C. acknowledges financial support from the Horizon 2020 Framework Programme (Marie Skłodowska-Curie Grant No. 838280). V. B., S. E., S. Kaskel thank the DFG (FOR 2433 MOF Switches, Project Nr. 279409724) for financial support. F. W., S. Kaskel acknowledge funding from the European Research Council (ERC) under the European Union's Horizon 2020 research and innovation programme (grant agreement No. 742743). V. B. acknowledges German Federal Ministry for Education and Research (BMBF Project No 05K19OD2) for financial support. B. L. F. acknowledges financial support of the Netherlands Ministry of Education, Culture and Science (Gravitation program 024.601035) and the European Research Council (ERC, advanced grant no. 694345).

\section{Author contributions}

S. Krause designed and coordinated the project and synthesized the ligands and MOF materials. S. Krause, VB, DMT, DW, and NG built the in situ PXRD setup. MW and VB performed single crystal analysis and structure refinement. S. Krause and VB performed in situ PXRD experiments and structure refinement from PXRD data. SE performed SEM Analysis. S. Krause and VB performed adsorption experiments. S. Krause and FW performed in situ DRIFT and UV-Vis experiments. S. Krause and WD performed in situ characterization of AB photo switch. JDE performed MD and GCMC simulations. SC performed DFT simulations. WD and WB performed Raman experiments. S. Kaskel and BLF supported coordination of the project and writing of the manuscript. All Authors have read and contributed to the manuscript and supporting information.

\section{Data and materials availability:}

Crystal structure of DUT-163op and (E)-DUT-163-cp are available at the CCDC database under CCDC-2040810 and CCDC-2040811, respectively. Additional experimental studies to support the findings of this manuscript are displayed in the supplementary information or attached as additional supplementary information files. Raw data is either provided in the supplementary information can be obtained upon request from the corresponding authors.

\section{Conflict of interests:}

There are no conflicts to declare. 


\section{References}

$1 \quad$ Molecular Switches. 2 edn, Vol. 1,2 (Wiley-VCH Verlag GmbH \& Co. KGaA 2011).

2 Hugel, T. et al. Single-Molecule Optomechanical Cycle. Science 296, 1103-1106, doi:10.1126/science.1069856 (2002).

3 Liu, J. et al. Smart covalent organic networks (CONs) with "on-off-on" light-switchable pores for molecular separation. Sci. Adv. 6, eabb3188, doi:10.1126/sciadv.abb3188 (2020).

4 Hosono, N. et al. Large-Area Three-Dimensional Molecular Ordering of a Polymer Brush by One-Step Processing. Science 330, 808-811, doi:10.1126/science.1195302 (2010).

5 Ikeda, T. \& Tsutsumi, O. Optical Switching and Image Storage by Means of Azobenzene Liquid-Crystal Films. Science 268, 1873-1875, doi:10.1126/science.268.5219.1873 (1995).

6 Rice, A. M. et al. Photophysics Modulation in Photoswitchable Metal-Organic Frameworks. Chem. Rev. 120, 8790-8813, doi:10.1021/acs.chemrev.9b00350 (2020).

7 Haldar, R., Heinke, L. \& Wöll, C. Advanced Photoresponsive Materials Using the MetalOrganic Framework Approach. Adv. Mater. 32, 1905227, doi:10.1002/adma.201905227 (2020).

8 Jones, C. L., Tansell, A. J. \& Easun, T. L. The lighter side of MOFs: structurally photoresponsive metal-organic frameworks. J. Mater. Chem. A 4, 6714-6723, doi:10.1039/C5TA09424K (2016).

9 Kanj, A. B., Müller, K. \& Heinke, L. Stimuli-Responsive Metal-Organic Frameworks with Photoswitchable Azobenzene Side Groups. Macromol. Rapid Commun. 39, 1700239, doi:10.1002/marc.201700239 (2018).

10 Castellanos, S., Kapteijn, F. \& Gascon, J. Photoswitchable metal organic frameworks: turn on the lights and close the windows. CrystEngComm 18, 4006-4012, doi:10.1039/C5CE02543E (2016).

11 Horike, S., Shimomura, S. \& Kitagawa, S. Soft porous crystals. Nat. Chem. 1, 695, doi:10.1038/nchem.444 (2009).

12 Li, D. \& Kaneko, K. Hydrogen bond-regulated microporous nature of copper complexassembled microcrystals. Chem. Phys. Lett. 335, 50-56, doi:10.1016/S0009-2614(00)01419-6 (2001).

13 Loiseau, T. et al. A Rationale for the Large Breathing of the Porous Aluminum Terephthalate (MIL-53) Upon Hydration. Chem. Eur. J. 10, 1373-1382, doi:10.1002/chem.200305413 (2004).

14 Krause, S. et al. A pressure-amplifying framework material with negative gas adsorption transitions. Nature 532, 348-352, doi:10.1038/nature17430 (2016).

15 Gu, C. et al. Design and control of gas diffusion process in a nanoporous soft crystal. Science 363, 387-391, doi:10.1126/science.aar6833 (2019).

16 Sato, H. et al. Self-Accelerating CO Sorption in a Soft Nanoporous Crystal. Science 343, 167170, doi:10.1126/science.1246423 (2014).

17 Mason, J. A. et al. Methane storage in flexible metal-organic frameworks with intrinsic thermal management. Nature 527, 357, doi:10.1038/nature15732 (2015).

18 Krause, S., Hosono, N. \& Kitagawa, S. Chemistry of Soft Porous Crystals: Structural Dynamics and Gas Adsorption Properties. Angew. Chem. Int. Ed. 59, 15325-15341, doi:10.1002/anie.202004535 (2020).

19 Schneemann, A. et al. Flexible metal-organic frameworks. Chem. Soc. Rev. 43, 6062-6096, doi:10.1039/C4CS00101J (2014).

20 Lee, J. H., Jeoung, S., Chung, Y. G. \& Moon, H. R. Elucidation of flexible metal-organic frameworks: Research progresses and recent developments. Coord. Chem. Rev. 389, 161-188, doi:https://doi.org/10.1016/j.ccr.2019.03.008 (2019).

21 Zheng, Y. et al. Flexible interlocked porous frameworks allow quantitative photoisomerization in a crystalline solid. Nat. Commun. 8, 100, doi:10.1038/s41467-017-00122-5 (2017).

22 Nikolayenko, V. I., Herbert, S. A. \& Barbour, L. J. Reversible structural switching of a metalorganic framework by photoirradiation. Chem. Commun. 53, 11142-11145, doi:10.1039/C7CC06074B (2017).

23 Tan, P., Jiang, Y., Liu, X.-Q. \& Sun, L.-B. Making Porous Materials Respond to Visible Light. ACS Energy Lett. 4, 2656-2667, doi:10.1021/acsenergylett.9b01970 (2019). 
24 Huang, S.-L., Hor, T. S. A. \& Jin, G.-X. Photodriven single-crystal-to-single-crystal transformation. Coord. Chem. Rev. 346, 112-122, doi:https://doi.org/10.1016/j.ccr.2016.06.009 (2017).

25 Lyndon, R. et al. Dynamic Photo-Switching in Metal-Organic Frameworks as a Route to Low-Energy Carbon Dioxide Capture and Release. Angew. Chem. Int. Ed. 52, 3695-3698, doi:doi:10.1002/anie.201206359 (2013).

26 Schaate, A. et al. A Novel Zr-Based Porous Coordination Polymer Containing Azobenzenedicarboxylate as a Linker. Eur. J. Inorg. Chem. 2012, 790-796, doi:10.1002/ejic.201101151 (2012).

27 Das, G. et al. Azobenzene-Equipped Covalent Organic Framework: Light-Operated Reservoir. J. Am. Chem. Soc. 141, 19078-19087, doi:10.1021/jacs.9b09643 (2019).

28 Khayyami, A., Philip, A. \& Karppinen, M. Atomic/Molecular Layer Deposited IronAzobenzene Framework Thin Films for Stimuli-Induced Gas Molecule Capture/Release. Angew. Chem. Int. Ed. 58, 13400-13404, doi:10.1002/anie.201908164 (2019).

29 Epley, C. C. et al. Cargo delivery on demand from photodegradable MOF nano-cages. Dalton Trans. 46, 4917-4922, doi:10.1039/C6DT04787D (2017).

30 Roth Stefaniak, K. et al. Photo-triggered release of 5-fluorouracil from a MOF drug delivery vehicle. Chem. Commun. 54, 7617-7620, doi:10.1039/C8CC01601A (2018).

31 Stoeck, U., Krause, S., Bon, V., Senkovska, I. \& Kaskel, S. A highly porous metal-organic framework, constructed from a cuboctahedral super-molecular building block, with exceptionally high methane uptake. Chem. Commun. 48, 10841-10843, doi:10.1039/C2CC34840C (2012).

32 Evans, J. D., Bocquet, L. \& Coudert, F.-X. Origins of Negative Gas Adsorption. Chem 1, 873886, doi:10.1016/j.chempr.2016.11.004 (2016).

33 Crespi, S., Simeth, N. A. \& König, B. Heteroaryl azo dyes as molecular photoswitches. Nat. Rev. Chem. 3, 133-146, doi:10.1038/s41570-019-0074-6 (2019).

34 Dokić, J. et al. Quantum Chemical Investigation of Thermal Cis-to-Trans Isomerization of Azobenzene Derivatives: Substituent Effects, Solvent Effects, and Comparison to Experimental Data. J. Phys. Chem. A 113, 6763-6773, doi:10.1021/jp9021344 (2009).

35 Evans, J. D., Krause, S., Kaskel, S., Sweatman, M. B. \& Sarkisov, L. Exploring the thermodynamic criteria for responsive adsorption processes. Chem. Sci. 10, 5011-5017, doi:10.1039/C9SC01299K (2019).

36 Stuart, C. M., Frontiera, R. R. \& Mathies, R. A. Excited-State Structure and Dynamics of cisand trans-Azobenzene from Resonance Raman Intensity Analysis. J. Phys. Chem. A 111, 12072-12080, doi:10.1021/jp0751460 (2007).

37 Krause, S. et al. The effect of crystallite size on pressure amplification in switchable porous solids. Nat. Commun. 9, 1573, doi:10.1038/s41467-018-03979-2 (2018).

38 Tylkowski, B., Jastrząb, R. \& Skrobańska, M. Photo-sensitive complexes based on azobenzene. Phys. Sci. Rev. 1, 20160002, doi:https://doi.org/10.1515/psr-2016-0002 (2016).

39 Krause, S. et al. A pressure-amplifying framework material with negative gas adsorption transitions. Nature 532, 348-352, doi:10.1038/nature17430 (2016).

40 Krause, S. et al. Adsorption Contraction Mechanics: Understanding Breathing Energetics in Isoreticular Metal-Organic Frameworks. J. Phys. Chem. C 33, 19171-19179, doi:10.1021/acs.jpcc.8b04549 (2018).

41 Krause, S. et al. Towards general network architecture design criteria for negative gas adsorption transitions in ultraporous frameworks. Nat. Commun. 10, 3632, doi:10.1038/s41467-019-11565-3 (2019).

42 Krause, S. et al. Engineering micromechanics of soft porous crystals for negative gas adsorption. Chem. Sci. 11, 9468-9479, doi:10.1039/D0SC03727C (2020).

43 Krause, S. et al. The Role of Temperature and Adsorbate on Negative Gas Adsorption in the Mesoporous Metal-Organic Framework DUT-49. Faraday Discuss., doi:10.1039/D0FD00013B (2020).

44 Simeth, N. A., Bellisario, A., Crespi, S., Fagnoni, M. \& König, B. Substituent Effects on 3Arylazoindole Photoswitches. J. Org. Chem. 84, 6565-6575, doi:10.1021/acs.joc.8b02973 (2019). 
45 Bandara, H. M. D. \& Burdette, S. C. Photoisomerization in different classes of azobenzene. Chem. Soc. Rev. 41, 1809-1825, doi:10.1039/C1CS15179G (2012).

46 Stoychev, G., Kirillova, A. \& Ionov, L. Light-Responsive Shape-Changing Polymers. Adv. Opt. Mater. 7, 1900067, doi:10.1002/adom.201900067 (2019). 
